\author{
Nota Científica \\ (Short Communication)
}

\title{
LA GUACAMAYA VERDE (ARA MILITARIS) EN LA SIERRA GORDA DE GUANAJUATO, MÉXICO
}

\author{
Recibido: 27/05/2014; aceptado: 14/03/2016
}

Ramírez-Albores, J. E. 2016. La Guacamaya verde (Ara militaris) en la Sierra Gorda de Guanajuato, México. [Military macaw (Ara militaris) in Sierra Gorda, Guanajuato, Mexico]. Acta Zoológica Mexicana (n.s.), 32(2): 182-185.

\begin{abstract}
I report a new displacement route of the Military macaw (Ara militaris) population located in Sotano del Barro (Querétaro) to the northeastern Guanajuato. This record suggests that Military macaw perform seasonal movements in search of foraging areas between the Sotano del Barro in Xichú (Guanajuato), my study area. Therefore, the presence of Military macaw in the region would have direct implications for policy change in land use, which directly affects the land management of the Sierra Gorda Biosphere Reserve.
\end{abstract}

La Guacamaya verde (Ara militaris) es una especie residente de la región neotropical que presenta una distribución discontinua desde México hasta el noreste de Argentina, ausentándose en Centroamérica (Forshaw 1989, Howell \& Webb 1995, Juniper \& Parr 1998). Esta guacamaya realiza movimientos diarios y estacionales entre diferentes tipos de vegetación (Forshaw 1989, Howell \& Webb 1995, Juniper \& Parr 1998; Iñigo-Elías 1999, 2000), dentro de un área relativamente restringida (Collar 1997, Botero-Delgadillo \& Páez 2011). Diversos estudios referentes a la Guacamaya verde en varias regiones han reportado fluctuaciones en su abundancia poblacional y movimientos de rutas de vuelo (Collar 1997, Gaucín 2000, Salazar 2001, Strewe \& Navarro 2003, Flórez \& Sierra 2004, Bonilla-Ruz et al. 2007ab, Arizmendi 2008, Navarro et al. 2008, Rivera-Ortiz et al. 2008, ContrerasGonzález et al. 2009), que parecen indicar movimientos migratorios o desplazamientos entre dormideros/nidos y sitios de forrajeo. En estos estudios se han registrado distancias máximas diarias de desplazamiento de $20 \mathrm{~km}$ entre los sitios de nidificación/dormideros y los sitios de alimentación (Gaucín 2000, Bonilla-Ruz et al. 2007b) con requerimientos de área entre 4989 a 12,309 ha (Boni1la-Ruz et al. 2007a).

En México, las poblaciones de Guacamaya verde también presentan una distribución discontinua (Howell
\& Webb 1995, Iñigo-Elías 2000), encontrando diversas poblaciones aisladas en la vertiente del Pacífico, desde Sonora y suroeste de Chihuahua hasta Oaxaca y Chiapas, y en la costa del Golfo desde Tamaulipas al centro de México en San Luis Potosí, Estado de México y Querétaro (Howell \& Webb 1995, Iñigo-Elías 2000). Sin embargo, en la última década se han incrementado los registros de este psitácido en varias regiones del país (e.g., Puebla, Guerrero, Morelos y Estado de México; UrbinaTorres et al. 2009, Hernández et al. 2012, Jiménez-Arcos et al. 2012, Urbina-Torres et al. 2012, Villaseñor \& Botello 2012). Para Guanajuato sólo existían reportes de individuos de Guacamaya verde en el cañón de Atarjea provenientes de la población ubicada en el Sótano del Barro en Querétaro (Gaucín 2000). Por tanto, como parte de la falta de conocimiento de la distribución completa de esta especie, este trabajo contribuye al conocimiento de la capacidad de desplazamiento de la Guacamaya verde documentando una nueva ruta de desplazamiento en Guanajuato de la población localizada en el Sótano del Barro en Querétaro.

El área de registro se encuentra en el Municipio de Xichú (Fig. 1), en el límite noreste de la Reserva de la Biosfera Sierra Gorda en Guanajuato (RBSG; Fig. 1). Esta reserva tiene una extensión de 236,882 ha, y fue decretada en febrero de 2007 (DOF 2007). El área de registro se encuentra adyacente a la Reserva de la Biosfera Sierra Gorda en Querétaro (Fig. 1). La altitud tiene un rango entre 900 y 2600 msnm y presenta dos tipos de clima, un clima semi-árido templado con lluvias en verano, y un clima semi-árido cálido con lluvias en verano. La precipitación anual varía de $500 \mathrm{~mm}$ (en climas áridos) a 2500 $\mathrm{mm}$ (en climas húmedos), y la temperatura media anual tiene un rango entre $12^{\circ}$ a $22{ }^{\circ} \mathrm{C}$ (CONANP 2005, Secretaría de Gobernación 2007). La vegetación está dominada por bosque tropical caducifolio en el cauce inferior adyacente al río Santa María, así como en las cañadas que lo rodean, con dominancia de especies como Bursera morelensis, Cordia boissierii, Fouquierria splendens, Ceiba 


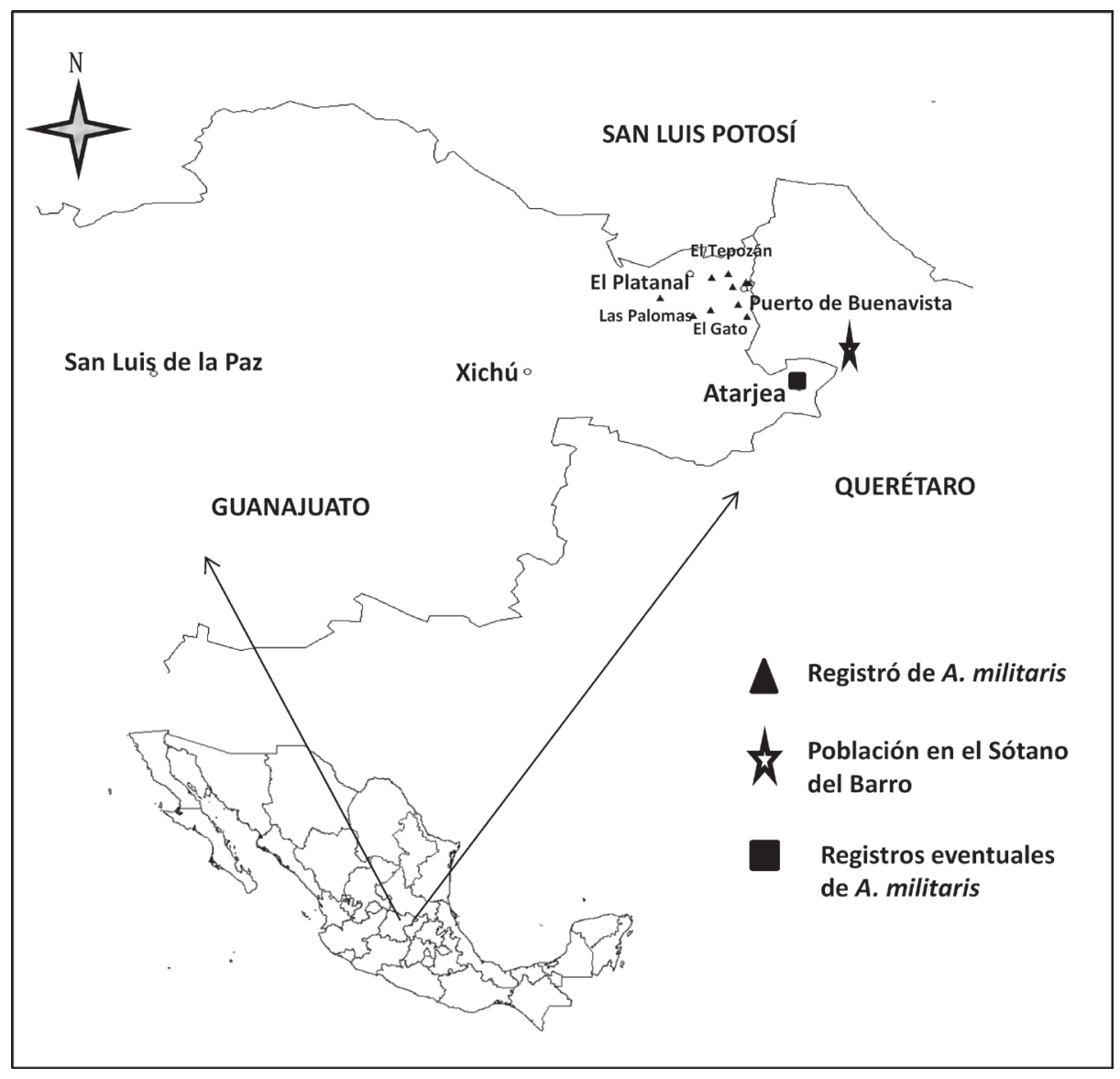

Figura 1. Ubicación del área de registro de Guacamaya verde (Ara militaris) en la Sierra Gorda de Guanajuato, México. Localidades de referencia de Guacamaya verde en la región $(\circ)$, sitios de registro $(\boldsymbol{\Delta})$, y registros previos de la población en el Sótano del Barro en Querétaro

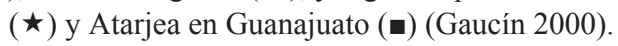

spp., Ipomoea spp., y Stenocereus queretaroensis (DOF 2007). En las partes altas, domina el matorral submontano y bosque de pino-encino (Carranza 2005), donde las especies vegetales dominantes son Fouquierria splendens, Prosopis laevigata, Ipomoea spp., Pinus michoacana, P. teocote, Juniperus flaccida, Quercus laurina, $Q$. affinis, $Q$. castanea, $Q$. crassifolia y $Q$. rugosa (Carranza 2005).

Durante noviembre de 2011 a agosto de 2012 se realizaron registros ocasionales en que se observaron grupos de 10 a 22 individuos de Guacamaya verde en diferentes sitios del Municipio de Xichú, Guanajuato $\left(21^{\circ} 25^{\prime}\right.$ N, $99^{\circ} 47^{\prime} \mathrm{W} ; 21^{\circ} 24^{\prime} \mathrm{N}, 99^{\circ} 53^{\prime} \mathrm{W}$; Fig. 1). El 5 de noviembre de 2011, se observó un grupo de 22 individuos sobrevolando y emitiendo vocalizaciones en una cañada con bosque de pino-encino (21 $23^{\prime} \mathrm{N}, 99^{\circ} 47^{\prime} \mathrm{W}$ a 1627 msnm) a $3 \mathrm{~km}$ SO de la localidad de Puerto de Buenavista (entre las 9:00-10:00 h). Este grupo sobrevoló de la parte noroeste a lo largo de las cañadas de bosque de pino-encino y áreas adyacentes, hacia cañadas con bosque caducifolio y con vegetación riparia. El sobrevuelo del grupo de guacamayas se dirigió en dirección de la localidad de El Platanal, los cerros de la Mina y del Espíritu Santo y cañadas adyacentes al río Santa María; algunos de los individuos (3-4) percharon durante $10 \mathrm{~min}$ en árboles de Bursera spp. y Juglans mollis. El 25 de diciembre de 2011 (entre 16:00 a 17:00 h), 10 individuos fueron obser-

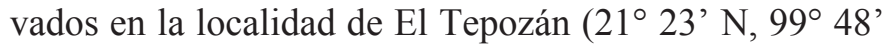
W a $1651 \mathrm{msnm}$ ), y dos individuos de este grupo percharon en un encino (Quercus spp.) durante 5 min, después volaron en dirección sureste para unirse al resto del grupo (T. Olvera, com. pers.). El 14 de enero de 2012, se observó un grupo de 12 individuos sobrevolando y emitiendo vocalizaciones en una cañada con bosque de pino-encino cercana a la localidad de El Gato (entre 10:00-11:00 h) a $1.3 \mathrm{~km}$ al este $\left(21^{\circ} 23^{\prime} \mathrm{N}, 99^{\circ} 48^{\prime} \mathrm{W}\right.$ a $\left.1651 \mathrm{msnm}\right)$. 
Sesenta minutos más tarde, se observó este mismo grupo sobrevolando cerca de la localidad de Las Palomas $\left(21^{\circ}\right.$ 22' N, 99 50' W a 1272 msnm; Fig. 1). Después, este grupo se dirigió probablemente en dirección norte a áreas cubiertas por bosque caducifolio y vegetación riparia cercanas al río Santa María. En este mismo mes, se observó un grupo de 14 individuos sobrevolando las localidades de Las Palomas (21 ${ }^{\circ} 22^{\prime}$ N, $99^{\circ} 51^{\prime}$ W a 1269 msnm) y El Pinalito $\left(21^{\circ} 24^{\prime} \mathrm{N}, 99^{\circ}\right.$ 53' W a 1795 msnm; T. Olvera com. pers.). El 6 de junio de 2012 (entre 09:40-10:30 hrs) otro grupo de 12 individuos fue observado sobrevolando la localidad de El Roblar; volaban en dirección sureste. Finalmente, en agosto de 2013 se realizó un monitoreo durante cinco días consecutivos en los mismos lugares donde fue registrada anteriormente la Guacamaya verde. Durante estos días, no hubo registro alguno de individuos de Guacamaya verde, y los pobladores locales de algunas localidades comentaron que no la habían observado en más de un mes.

Los individuos registrados de Guacamaya verde sobrevuelan diversas zonas en busca de sitios de percha y forrajeo en la parte oeste de la región, principalmente en áreas de bosque de pino-encino, bosque caducifolio y vegetación riparia en dirección al río Santa María y a los cerros de la Mina y del Espíritu Santo. Estos grupos observados de guacamaya en Guanajuato, es probable que provengan del Sótano de Barro en Querétaro (a $20 \mathrm{~km}$ al suroeste de la zona de estudio), que es la población residente más cercana (50-80 individuos), y donde se han identificado al menos cinco rutas de desplazamiento de hasta $21 \mathrm{~km}$, nidos activos y áreas de alimentación (Gaucín 2000). Sin embargo, cuatro de estas rutas de desplazamiento (entre 3$7 \mathrm{~km}$ ) se realizan en la región del Sótano del Barro dentro de los municipios de Arroyo Seco y Jalpan en Querétaro, y solo una es en Guanajuato (en el cañón de Atarjea a 6 $\mathrm{km}$ al suroeste del Sótano de Barro). Siendo esta última, el registro previo más cercano de guacamaya en Guanajuato (a $15 \mathrm{~km}$ al sureste de la zona de estudio); sin embargo, estos registros fueron eventuales y no se registraron sitios de anidación y forrajeo (Gaucín 2000). Cabe mencionar, que estos dos sitios cuentan con una vegetación similar a la del área de estudio pero presentan un mayor grado de perturbación.

La presencia de la Guacamaya verde en la Sierra Gorda de Guanajuato amplía el conocimiento del área de distribución conocida de la población del Sótano del Barro en Querétaro como una nueva ruta de desplazamiento a $20 \mathrm{~km}$ al noroeste en dirección al municipio de Xichú. Estos registros de guacamaya en esta área junto con los comentarios de los pobladores locales, sugieren de forma preliminar que sólo realizan movimientos estacionales en busca de sitios de forrajeo entre el Sótano del Barro (Querétaro) y el área de registro en el municipio de Xichú en Guanajuato, ausentándose entre los meses de mayo a octubre. A la fecha aún no se tiene registro de sitios de anidación y reproducción. Dada la relevancia de las observaciones de una especie importante para la conservación tanto a nivel regional como global (SEMARNAT 2010, BirdLife International 2013), se propone extender la superficie en $20 \mathrm{~km}$ al sureste del área núcleo de la RBSG en Guanajuato, abarcando los sitios de registro y el cañón de Atarjea como ruta de desplazamiento de la Guacamaya verde. Por tanto, la presencia de la Guacamaya verde en la región tendría implicaciones directas sobre las políticas de cambio de uso de suelo, lo cual debería incluir una extensión hacia el sur la superficie de esta reserva, incidiendo directamente en el ordenamiento ecológico territorial de la RBSG. Aunado a lo anterior, cabe señalar la relevancia de tener varias poblaciones pequeñas alrededor o junto a aquellas poblaciones más grandes o conocidas desde una perspectiva poblacional, con lo que se deben verificar aspectos demográficos como la migración y la colonización. Esta perspectiva permite sugerir estudios a futuro para determinar el origen y la conectividad de las poblaciones a través de marcaje de individuos $\mathrm{o}$ análisis genéticos. Finalmente, el escaso conocimiento de la biodiversidad de Guanajuato, nos permite inferir que todavía existen lagunas importantes en el conocimiento de la distribución actual de la Guacamaya verde, así como de su biología y ecología. Por ello, se deben de aumentar los esfuerzos en el monitoreo de la avifauna de muchas regiones del país, para tener un mayor y mejor conocimiento de su biodiversidad.

AGRADECIMIENTOS. A C. Vázquez, L. del Villar, J.L. Medina y M. Pérez por la asistencia en campo. A T. Olvera y a la familia Velázquez-González por su ayuda y apoyo durante el trabajo de campo. Comentarios y sugerencias de R. Rodríguez-Estrella y dos revisores anónimos mejoraron este manuscrito. A A. Navarro-Sigüenza por la elaboración del mapa de distribución.

\section{LITERATURA CITADA}

Arizmendi, M. C. 2008. Conservación de la Guacamaya verde (Ara militaris) en la Reserva de la Biosfera Tehuacán-Cuicatlán, México: un estudio de abundancia y reproducción en la zona de la Cañada. Facultad de Estudios Superiores Iztacala, Universidad Nacional Autónoma de México, México, D.F. 
BirdLife International. 2013. Ara militaris. The IUCN Red List of Threatened Species version 2015.2. Available at http://www.iucnredlist.org (accessed on August 2015).

Bonilla-Ruz, C., Reyes, G. \& Cruz, L. S. 2007a. Ámbito hogareño de la Guacamaya Verde (Ara militaris) en la cañada Oaxaqueña. Mesoamericana, 11: 53-59.

Bonilla-Ruz, C., Reyes, G. \& García, R. 2007b. Observations of Military Macaw (Ara militaris) in northern Oaxaca, Mexico. Wilson Journal of Ornithology, 119: 731-734.

Botero-Delgadillo, E. \& Páez, C. A. 2011. Estado actual del conocimiento y conservación de los loros amenazados de Colombia. Conservación Colombiana, 14: 86-151.

Carranza, E. 2005. Conocimiento actual de la flora y la diversidad vegetal del estado de Guanajuato, México. Pp. 17. In: J. Rzedowsky \& G. Calderón de Rzedowsky (Eds.). Flora del Bajío y de Regiones Adyacentes. Fascículo Complementario 21. México.

Collar, N. J. 1997. Family Psittacidae (parrots). pp. 280-477. In: J. Del Hoyo, A. Elliot \& J. Sargatal (Eds.). Handbook of the birds of the world. Volume 4. Sandgrouse to cuckoos. Lynx Eds., Barcelona.

CONANP (Comisión Nacional de Áreas Naturales Protegidas). 2005. Estudio previo justificativo para el establecimiento del área natural protegida "Reserva de la Biosfera Sierra Gorda de Guanajuato". CONANP-SEMARNAT. México.

Contreras-González, A. M., Rivera-Ortiz, F. A., Soberanes-González, C., Valiente-Banuet, A. \& Arizmendi, M. C. 2009. Feeding ecology of Military Macaws (Ara militaris) in a semi-arid region of central México. Wilson Journal of Ornithology, 121:384-391.

DOF (Diario Oficial de la Federación). 2007. Decreto por el que se declara área natural protegida, con el carácter de reserva de la biosfera, la zona conocida como Sierra Gorda de Guanajuato localizada en los municipios de Atarjea, San Luis de la Paz, Santa Catarina, Victoria y Xichú, en el Estado de Guanajuato. Diario Oficial de la Federación. Primera Sección. Viernes 2 de Febrero del 2007: 25-46.

Flórez, P. \& Sierra, A. 2004. Iniciativa para la conservación de la Guacamaya verde (Ara militaris) y su hábitat en el occidente de Antioquia, Colombia. Fundación Omacha-Proaves, Bogotá.

Forshaw, J. M. 1989. Parrots of the world. Lansdowne Eds. Melbourne, Australia.

Gaucín R., N. 2000. Biología de la conservación de la Guacamaya verde (Ara militaris) en el Sótano del Barro, Querétaro. Universidad Autónoma de Querétaro. Facultad de Ciencias Naturales. Informe final SNIB-CONABIO proyecto No. L204. México D.F.

Hernández C., J., Villordo, J. A., Cano, B. I., Gaspariano, E. \& Rodríguez, P. 2012. Registro de Guacamaya verde (Ara militaris) en la UMA Santa Cruz Achichipilco, municipio de Teotlalco, Puebla, México. Huitzil, 13: 130-136.

Howell, G. S. N. \& Webb, S. 1995. A guide to the birds of Mexico and Northern Central America. Oxford University Press. New York.

Iñigo-Elías, E. 1999. Las guacamayas verde y escarlata en México. Biodiversitas, 25: 7-11.

Iñigo-Elías, E. 2000. Guacamaya verde (Ara militaris). Pp. 213-215. In: G. Ceballos \& L. Márquez (Coords.). Las aves de México en peligro de extinción. Fondo de Cultura Económica. México.
Jiménez-Arcos, V. H., Santa, S. A., Escalona, A., Arizmendi, M. C. \& Vázquez, L. 2012. Ampliación de la distribución y presencia de una colonia reproductiva de la Guacamaya verde (Ara militaris) en el Alto Balsas de Guerrero, México. Revista Mexicana de Biodiversidad, 83: 864-867.

Juniper, T. \& Parr, M. 1998. Parrots. A guide to parrots of the world. Yale University Press. London, UK.

Navarro, M. E., Gallegos, M. O., Garay, D. B., Ortiz, B. F., Cueva, M. \& Rodríguez, L. 2008. Registro de una población de Guacamayo Verde Ara militaris (Linnaeus, 1766) en el departamento General San Martín, provincia de Salta, Argentina y consideraciones para su conservación. Nótulas Faunísticas, Segunda Serie, 22:1-11.

Rivera-Ortiz, F. A., Contreras, A. M., Soberanes, C. A., Valiente, A. \& Arizmendi, M. C. 2008. Seasonal abundance and breeding chronology of the military macaw (Ara militaris) in a semi-arid region of Central Mexico. Ornitología Neotropical, 19: 255-263.

Salazar T., J. M., Bonilla-Ruiz, C. \& Ximello-Olguín, F. X. 2010. Primer Registro de Guacamaya Verde (Ara militaris) en el municipio de Tehuacán, Puebla, México. Mesoamericana, 14: 59-66.

Secretaría de Gobernación. 2007. Decreto por el que se declara área natural protegida, con el carácter de reserva de la biosfera, la zona conocida como Sierra Gorda de Guanajuato localizada en los municipios de Atarjea, San Luis de la Paz, Santa Catarina, Victoria y Xichú en el Estado de Guanajuato. Diario Oficial de la Federación 2 de febrero del 2007:1- 47.

SEMARNAT (Secretaría del Medio Ambiente y Recursos Naturales). 2010. Norma Oficial Mexicana NOM-059-SEMARNAT-2010, protección ambiental-especies nativas de México de flora y fauna silvestres-categorías de riesgo y especificaciones para su inclusión, exclusión o cambio-lista de especies en riesgo. Diario Oficial de la Federación 30 de diciembre del 2010:1-78.

Strewe, R. \& Navarro, C. 2003. New distributional records and conservation importance of the San Salvador Valley, Sierra Nevada de Santa Marta, northern Colombia. Ornitología Colombiana, 1: $28-40$.

Urbina-Torres, F., Romo de Vivar-Álvarez, C. \& Navarro, A. G. 2009. Notas sobre la distribución de algunas aves en Morelos, México. Huitzil, 10: 30-37.

Urbina-Torres, F., Monroy, O., González, A., Amador, C. L. R. \& Celis, A. 2012. Nuevos registros de aves para el estado de México. Revista Mexicana de Biodiversidad, 83: 1241-1243.

Villaseñor, E. \& Botello, F. 2012. Registros notables de la Guacamaya verde (Ara militaris) en el norte del estado de Guerrero, México. Acta Zoológica Mexicana (n.s.), 28: 465-470.

\section{JORGE E. RAMÍREZ-ALBORES}

Instituto Potosino de Investigación Científica y Tecnológica, A. C. Camino a la Presa San José 2055, Col. Lomas 4a. Sección, 78216. San Luis Potosí, San Luis Potosí, México.<jorgeramirez22@hotmail. com> 